\title{
A Perspective on the Performance of the CFOSAT Rotating Fan-Beam Scatterometer
}

\author{
Wenming Lin ${ }^{\circledR}$, Senior Member, IEEE, Xiaolong Dong, Senior Member, IEEE, Marcos Portabella ${ }^{\circledR}$, \\ Shuyan Lang, Yijun $\mathrm{He}^{\circledR}$, Member, IEEE, Risheng Yun, Zhixiong Wang, \\ $\mathrm{Xingou} \mathrm{Xu}^{\circledR}$, Member, IEEE, Di Zhu, Member, IEEE, and Jianqiang Liu
}

\begin{abstract}
The China-France Oceanography Satellite (CFOSAT) to be launched in October 2018 will carry two innovative payloads, i.e., the surface wave investigation and monitoring instrument and the rotating fan-beam scatterometer [CFOSAT scatterometer (CFOSCAT)]. Both instruments, operated in $\mathrm{Ku}$-band microwave frequency, are dedicated to the measurement of sea surface wave spectra and wind vectors, respectively. This paper provides an overview of the system definition and characteristics of the CFOSCAT instrument. A prelaunch analysis is carried out to estimate the scatterometer backscatter and wind quality based on the developed CFOSCAT simulator prototype. The overall simulation includes two parts: first, a forward model is developed to simulate the ocean backscatter signals, accounting for both instrument and geophysical noise. Second, a wind inversion processor is used to retrieve wind vectors from the outputs of the forward model. The benefits and challenges of the novel observing geometries are addressed in terms of the CFOSCAT wind retrieval. The simulations show that the backscatter accuracy and the retrieved wind quality of CFOSCAT are quite promising and meet the CFOSAT mission requirements.
\end{abstract}

Index Terms-Backscatter, inversion, measurement errors, remote sensing, rotating fan-beam scatterometer, simulation, wind.

\section{INTRODUCTION}

$\mathbf{O}$ VER the last four decades, considerable development of wind scatterometers has been carried out. All the

Manuscript received April 9, 2018; revised June 23, 2018 and July 11, 2018; accepted July 19, 2018. This work was supported in part by the National Natural Science Foundation of China under Grant 41706197 and in part by the Startup Foundation for Introducing Talent of NUIST. (Corresponding authors: Wenming Lin; Xiaolong Dong.)

W. Lin, Y. He, and Z. Wang are with the School of Marine Sciences, Nanjing University of Information Science and Technology, Nanjing 210044, China (e-mail: wenminglin@nuist.edu.cn; yjhe@nuist.edu.cn; wangzhixiong@ nuist.edu.cn).

X. Dong is with the CAS Key Laboratory of Microwave Remote Sensing, National Space Science Center, Chinese Academy of Sciences, Beijing 100190, China, and also with the School of Electronic, Electrical and Communication Engineering, University of Chinese Academy of Sciences, Beijing 100049, China (e-mail: dongxiaolong@mirslab.cn).

M. Portabella is with the Institut de Ciències del Mar (ICM-CSIC), 08003 Barcelona, Spain (e-mail: portabella@icm.csic.es).

S. Lang and J. Liu are with the National Ocean Satellite Application Center, Beijing 100081, China (e-mail: langshuyan@mail.nsoas.org.cn; liujianqiang@mail.nsoas.org.cn).

R. Yun, X. Xu, and D. Zhu are with the CAS Key Laboratory of Microwave Remote Sensing, National Space Science Center, Chinese Academy of Sciences, Beijing, China (e-mail: yunrisheng@mirslab.cn; xuxingou@mirslab.cn; zhudi@mirslab.cn).

Color versions of one or more of the figures in this paper are available online at http://ieeexplore.ieee.org.

Digital Object Identifier 10.1109/TGRS.2018.2858852 past and current spaceborne scatterometers can be classified into two different types, i.e., the fan-beam scatterometers with multiple fixed antenna orientation and the pencil-beam scatterometers with conically scanning antennas, thus resulting in varying viewing geometry. The former illuminates all wind vector cells (WVCs) across the subsatellite track at a set of fixed number of views and azimuth angles, while for the latter, the number of views and the azimuth angles vary with the WVC number or subsatellite cross-track position. The first fixed fan-beam scatterometer onboard SEASAT-A, i.e., the SEASAT-A Scatterometer System (SASS), was launched in 1978 and only operated for a few months [1]. As a follow-on design, the National Aeronautics and Space Administration (NASA) scatterometer (NSCAT) onboard the Japanese ADEOS-I satellite flew from August 1996 to June 1997 [2], [3]. Both SASS and NSCAT operated in Ku-band frequency and used Doppler filtering in the receiver for resolving the surface cells along the antenna footprints. Several C-band fixed fan-beam scatterometers have also been flown in the past, i.e., the Advanced Microwave Instrument onboard the two European Remote Sensing (ERS) satellites [4]. Moreover, the follow-on Advanced Scatterometers (ASCATs) onboard the MetOp satellite series are currently operating since 2006 (ASCAT-A) and 2012 (ASCAT-B) [5]. In particular, the ASCAT systems have been providing robust and consistent level-2 (L2) wind data products at various grid sampling intervals $(25$ and $12.5 \mathrm{~km})$ and backscatter $\left(\sigma^{0}\right)$ processing modes, i.e., nominal (Hamming window averaging) and coastal (box-car averaging) processing, for more than a decade [6]. The first rotating pencil-beam scatterometer, namely, SeaWinds onboard QuikSCAT, was launched in June 1999 [7], [8]. It provided high-quality wind data for more than 10 years until the failure of its spinning antenna in November 2009. A similar version of this instrument has been launched by various countries, such as the NASA SeaWinds- 2 onboard the Japanese ADEOS-2 satellite (December 2002October 2003), OSCAT onboard the Indian Oceansat- 2 satellite (September 2009-February 2014) [9], HSCAT on the Chinese HY-2A satellite (since August 2011) [10], the NASA RapidScat installed on the International Space Station (September 2014-August 2016) [11], and the Indian scatterometer satellite (SCATSAT-1, since September 2016) [12].

A novel concept of rotating scatterometer, namely, the rotating range-gated fan-beam scatterometer (RFSCAT), was first proposed through a feasibility study by the European Space 
Agency (ESA) in 2000 [13]. An end-to-end (E2E) simulator was then developed by the Royal Netherlands Meteorological Institute (KNMI) to evaluate the performances of a C-band RFSCAT with different system configurations [14]. Preliminary studies show that RFSCAT combines the characteristics of the fixed fan-beam scatterometer and the rotating pencil scatterometer, and can provide high-quality wind data [13], [14]. Following the pioneering simulations under the support of ESA, several RFSCAT projects have been carried out by China and Russia, including the Ku-band rotating fanbeam scatterometer on the China-France Oceanography Satellite (CFOSAT) [15], the dual-frequency (C- and Ku-bands) wind radar on the Chinese FengYun-3E satellite [16], and the Ku-band Russian scatterometer [17]. The CFOSAT scatterometer (CFOSCAT) flight model has been assembled and tested, and will be launched in October 2018, to become the first operational spaceborne RFSCAT system.

To date, the performances of all RFSCATs are evaluated by an E2E simulator. However, two essential elements of the simulator, i.e., the instrument characteristics and the measurement noise, have not been fully exploited yet. In this paper, a thorough prelaunch simulation is carried out in order to assess the potential benefits and challenges of CFOSCAT in terms of sea surface wind retrieval. The simulation includes a radar simulator developed on the basis of the defined instrument parameters, which can be used to estimate the radar-resolved spatial and radiometric resolution accurately. Section II overviews the CFOSCAT measurement concept and its system definition. Section III presents the detailed flowchart of the CFOSCAT simulator. In Section IV, some examples of simulated wind fields are illustrated, and performances on the retrieved wind quality are discussed. Finally, conclusions are summarized in Section V.

\section{SySTEM DEFINITION}

\section{A. Overview of the Instrument}

CFOSCAT is a Ku-band (central frequency of $13.256 \mathrm{GHz}$ ) real aperture radar, with one vertically $(\mathrm{V})$ polarized fan beam and one horizontally $(\mathrm{H})$ polarized fan-beam sweeping earth's surface at medium incidence angles $\left(\sim 26^{\circ}-51^{\circ}\right)$. The radar receives echoes with the same polarization as the emitted pulses, such that it actually has VV and $\mathrm{HH}$ polarized beams. Table I summarizes the main characteristics of the instrument parameters. These were defined as a result of various tradeoffs between the signal-to-noise ratio (SNR), the swath size, the platform constraints, and the wind performance requirements [18].

Fig. 1 (top) illustrates the 3-D view of the antenna architecture. It shows that the $\mathrm{VV}$ and $\mathrm{HH}$ beams are indeed offset by $180^{\circ}$ (see bottom), which is set to maximize the azimuth diversity of the observations acquired by the two different polarizations, with the objective of improving the retrieved wind quality [19]. In the nominal case, CFOSCAT transmits vertically and horizontally polarized pulses alternatively. Since one pulse cycle consists of $6.67 \mathrm{~ms}$, the pulse repetition rates of $\mathrm{VV}$ and $\mathrm{HH}$ beams are both $75 \mathrm{~Hz}$. The interval between each transmitted pulse and its corresponding echo
TABLE I

LIST OF THE MAIN CFOSCAT PARAMETERS

\begin{tabular}{|l|l|}
\hline Parameters & Values \\
\hline Transmitted power & $120 \mathrm{~W}$ \\
\hline Bandwidth & $0.5 \mathrm{MHz}$ \\
\hline Pulse duration & $1.35 \mathrm{~ms}$ \\
\hline $\begin{array}{l}\text { Pulse repetition rate } \\
\text { (PRF) }\end{array}$ & $75 \mathrm{~Hz}(\mathrm{VV})$ \\
\cline { 2 - 2 } Antenna peak gain & $75 \mathrm{~Hz}(\mathrm{HH})$ \\
\cline { 2 - 2 } & $32.0 \mathrm{~dB}(\mathrm{VV})$ \\
\hline \multirow{2}{*}{$\begin{array}{l}\text { One-way 3 dB beam } \\
\text { width }\end{array}$} & Range: $14.5^{\circ}$; Azimuth: $1.2^{\circ}(\mathrm{VV})$ \\
\cline { 2 - 2 } & Range: $15.0^{\circ} ;$ Azimuth: $1.1^{\circ}(\mathrm{HH})$ \\
\hline \multirow{4}{*}{ Antenna spinning rate } & $\begin{array}{l}\text { Default: } 3.4 \text { revolutions per } \text { minute } \\
\text { (rpm) }\end{array}$ \\
\cline { 2 - 2 } & High spinning rate: $3.74 \mathrm{rpm}$ \\
\cline { 2 - 2 } & Low spinning rate: $3.06 \mathrm{rpm}$ \\
\hline Power consumption & $150 \mathrm{~W}$ \\
\hline Instrument mass & $67 \mathrm{~kg}$ \\
\hline Swath & $1000 \mathrm{~km}$ \\
\hline
\end{tabular}

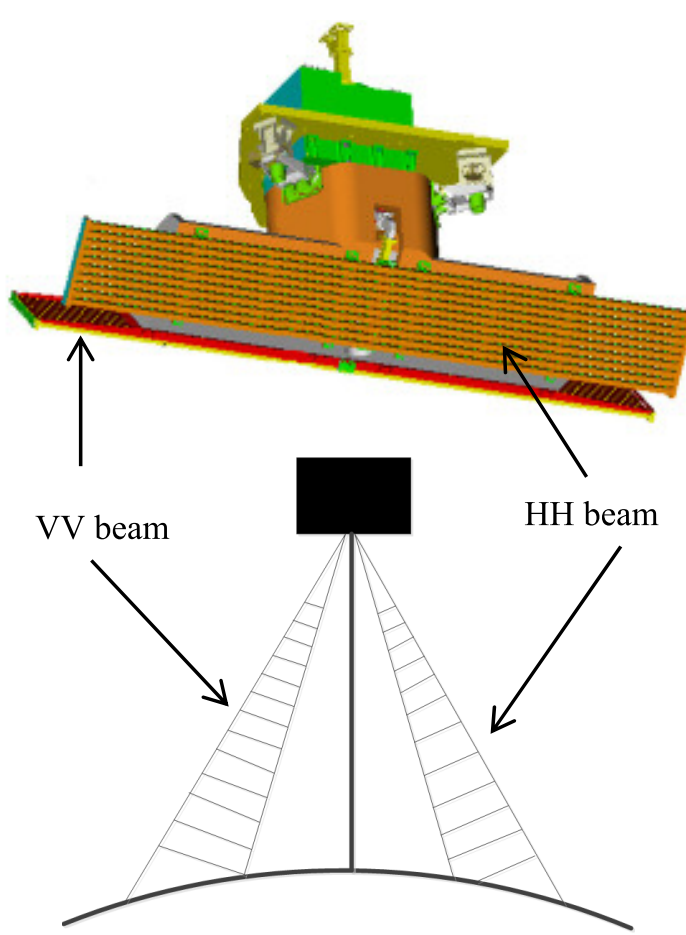

Fig. 1. (Top) 3-D model view of the antenna architecture of the CFOSAT scatterometer). (Bottom) Schematic illustration of the beams' configuration.

is about $3.8 \mathrm{~ms}$, which is exploited for internal calibration, thermal noise measurement, or passive measurement of brightness temperature (similar to radiometer). Fig. 2 shows the pulse timing scheme within one macrocycle, in which the number of internal calibration, radiometric measurement, and noise measurement is 1,8 , and 1 , respectively, for both $\mathrm{HH}$ and VV beams.

The transmitted pulse is written as [8]

$$
T(t)=\sqrt{E_{t}} p(t) e^{j 2 \pi\left(f_{c}+f_{d c}+\frac{1}{2} s \mu t\right) t}
$$

where $t$ is the time from the onset of each pulse, $E_{t}$ is the total emitted energy, $p(t)$ stands for the pulse power envelope 


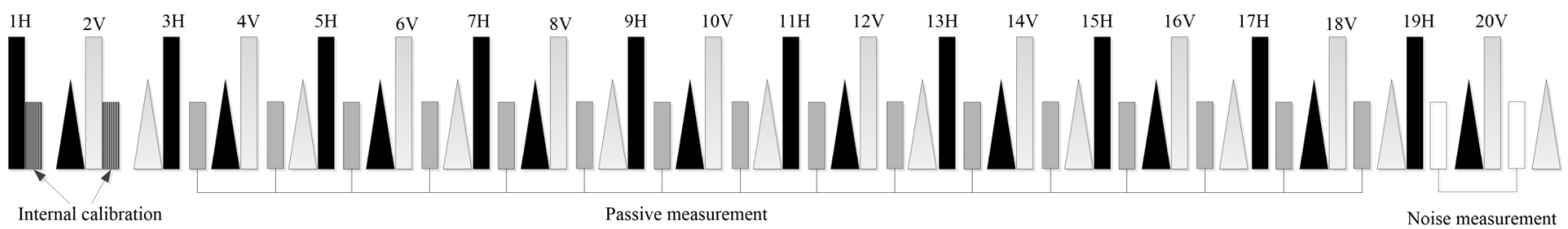

Fig. 2. Pulse timing scheme within one macrocycle. The long rectangles represent the transmitted pulse, and the short triangles stand for the received echoes. The measurement in the interval of each pulse cycle is with the same polarization as the emitted pulse.

such that $\int p^{2}(t) d t=1, f_{c}$ is the carrier frequency, $f_{d c}$ is the Doppler-compensation frequency, and $\mu=0.5 \mathrm{MHz} / 1.35 \mathrm{~ms}$ is the chirp rate of the linear-frequency-modulated pulse. In practice, the Doppler-compensation frequency $f_{d c}$ is defined as a function of beam azimuth angle, converted to a 1-D lookup table (LUT) with azimuth binning of $5.625^{\circ}$, and stored in a static Random Access Memory (RAM) of the CFOSCAT data processing unit. To ensure that the radar echo from the footprint is within the effective passband of the receiver, $f_{d c}$ may be updated by uploading a new version of the LUT to the satellite when the observing geometry changes remarkably.

Unlike other range-gated scatterometers, CFOSCAT includes an additional term $s$ in the transmitted signal, such that $s=1$ when the beam azimuth angle is within the range of $\left[90^{\circ}, 270^{\circ}\right)$, and $s=-1$ beyond this range. Here, the azimuth is defined in the satellite-fixed coordinate system, e.g., $0^{\circ}$ means forward looking (along the direction of satellite motion) and $180^{\circ}$ means backward looking. The additional term $s$ is implemented to avoid range ambiguity induced by the Doppler shift [18]. Similar to [8], the radar echo is digitally dechirped by mixing with a chirped reference signal, which converts range delays into frequency shifts, such that each discrete frequency in the dechirped signal corresponds to the return from a given surface area plus the corresponding Doppler shift. For CFOSCAT, the Doppler bandwidth associated with the large footprint is equivalent to the range-related signal bandwidth when the beam azimuth angle is close to $0^{\circ}$ or $180^{\circ}$, so the sign of these two terms is the same to avoid that two different surface areas appear in the same discrete frequency bin after dechirping.

\section{B. Onboard Processing}

The onboard processing is implemented to sample the backscatter signal in regular ground range gates and to reduce the overall data rate. This is achieved by the aforementioned dechirping technique, including a convolution of the received signal with a replica of the transmitted signal, a fast Fourier transformation (FFT, 4096 points) performed on the dechirped signal, and a squared-modulus detection applied on the FFT output. Due to the Doppler shift effect and the nonlinear relation between slant range and ground range (defined as the distance between satellite nadir point and the observed surface point), it is not straightforward to map the discrete FFT frequency bins to the regular ground range gates. Therefore, a 2-D LUT is designed to aggregate the FFT bins into 40 range gates (namely, slices in scatterometry). This LUT is a function of the beam azimuth and the indices of the range-gated (slice) boundary, with a dimension of 64 (beam azimuth) $\times 41$ (boundary indices). It is also stored in a RAM of the data processing unit, and can be updated during the operational stage. The number of FFT bins aggregated into slices slightly increases with the incidence angle. After integration, each slice has a surface range resolution of $\sim 10 \mathrm{~km}$, which is much lower than the intrinsic radar range resolution of $300 \mathrm{~m}$.

Assuming that the normalized radar cross section $\left(\sigma^{0}\right)$ is constant over a certain slice, the expected energy of the $i$ th slice is written as [8]

$$
E_{i}=X_{i} \cdot \sigma_{i}^{0}+E_{n}
$$

where $\sigma_{i}^{0}$ is the backscatter cross section corresponding to the $i$ th slice, $E_{n}$ is the noise energy, and $X_{i}$ is the calibration factor depicted as follows [8]:

$X_{i}=\frac{P_{t} G_{p}^{2} G_{r} \lambda^{2}}{(4 \pi)^{3} L} \sum_{j \in F} \frac{\Delta A_{j} g_{j}^{2}}{r_{j}^{4}} \sum_{k=k_{s}}^{k_{e}}\left\{\frac{\sin \left[\pi N_{j}\left(f_{b, j} T-\frac{k}{N}\right)\right]}{\sin \left[\pi\left(f_{b, j} T-\frac{k}{N}\right)\right]}\right\}^{2}$

where $P_{t}$ is the transmitted power, $G_{p}$ is the antenna peak gain, $G_{r}$ is the receiver gain, $L$ is the total two-way system loss, and $\lambda$ is the transmitted wavelength. The echo return from the surface can be modeled as the summation of backscatters from many independent surface patches within the footprint $F$, each with an area of $\Delta A_{j}$ and a distance to the receiver of $r_{j}$. Since the CFOSCAT antenna slowly rotates (Table I) and its azimuth beamwidth is $1.1^{\circ}-1.2^{\circ}$, the scanning loss, which is defined as the loss of signal power that occurs because of the shift of antenna gain pattern, is less than $0.05 \mathrm{~dB}$ [18]. Consequently, the normalized antenna gain in the direction of the $j$ th patch is assumed to be constant within the pulse cycle. Finally, $T$ is the sample period, $N$ is the FFT length, $k_{s}$ and $k_{e}$ are the start and end of FFT bin indices, $N_{j}$ is the length of the backscatter from the $j$ th patch captured by the range gate, and $f_{b, j}$ is the baseband frequency of the backscatter from the $j$ th patch, which is a combination of range delay and Doppler shift effect.

Note that the surface range dimension of CFOSCAT footprint is less than $250 \mathrm{~km}$, so the number of slices corresponding to the footprint is actually less than 25. Nonetheless, all the 40 slices are downloaded to the ground segment in order to capture the entire echo spectrum.

\section{Ground Processing}

The operational ground processing, to be developed in China by the National Ocean Satellite Application 


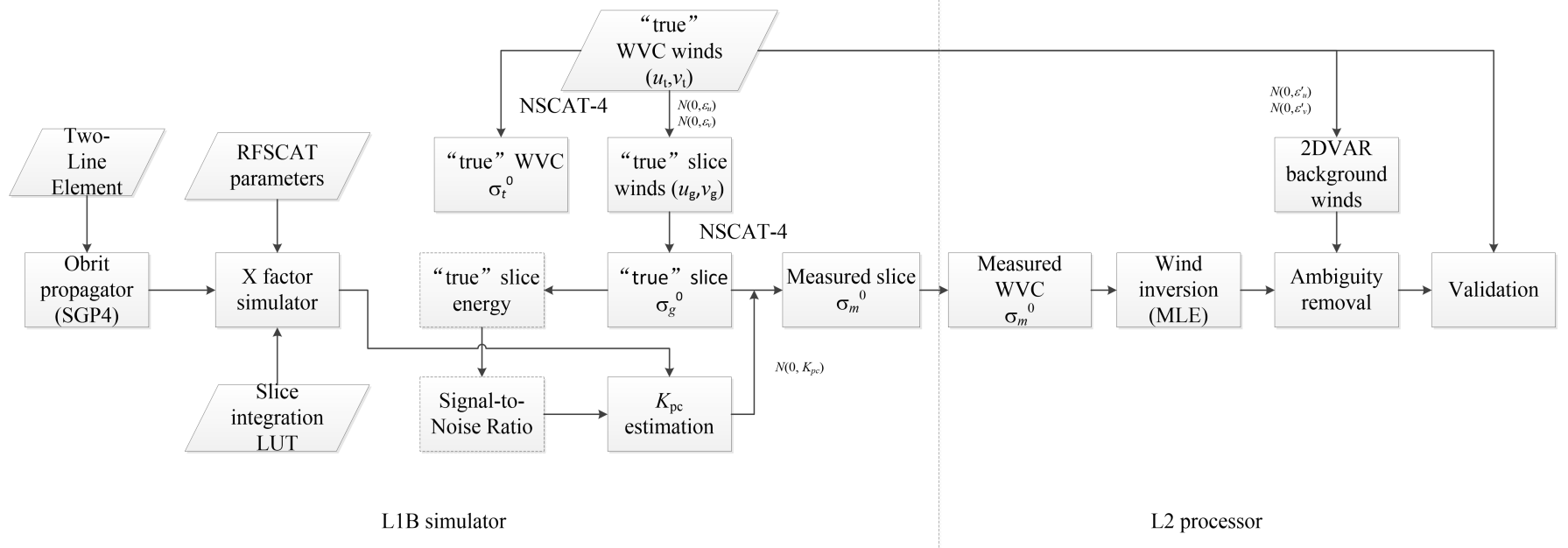

Fig. 3. Chart flow of the CFOSCAT prelaunch simulation. The left side of the dashed line represents the L1B simulator, while the right side of the dashed line represents the key steps of L2 processor. The dashed entries are only used to assess the geophysical noise or to simulate the instrument noise. The notations are explained in Section III.

Center (NSOAS), consists of several essential steps. At the so-called level-1 (L1) processing, the most relevant steps are: geolocation of the 40 slices (before any calibration), subtraction of a noise estimate from the raw signal value of each slice, correction of potential biases in the transmitted power and the inner system loss using the internal calibration signal, and correction of systematic effects in the antenna gain using external calibration with transponders or Amazon rainforest. Then, at the so-called L2 processing, the backscatter values with similar incidence and azimuth angles are averaged in a defined swath grid, and then wind retrieval is performed. The $\mathrm{L} 2$ processing generates the L2A product with averaged backscatter values and the L2B product with sea surface wind information. The produced L2A and L2B data files are both in the NetCDF format, and can be converted to L2 files (including backscatter values and wind fields) in Binary Universal Form for the Representation of meteorological data format by a complementary processing facility. Similar to the conventional scatterometer wind retrieval [20], [21], the L2 processing includes the following steps.

1) Slice Aggregation: Following convention and in order to minimize the computation requirements [21], the backscatter measurements that belong to the same WVC and correspond to the same antenna revolution are averaged into a particular WVC view. Note that the grid spacing is defined as 12.5 or $25 \mathrm{~km}$ before the averaging.

2) Wind Inversion: A set of averaged backscatter values in each WVC is inverted into a set of ambiguous wind solutions based on the commonly used inversion approach, i.e., the maximum likelihood estimator (MLE).

3) Ambiguity Removal (AR): A spatial filter, i.e., the so-called 2-D variational AR (2DVAR) approach [22], is then used to dealias the wind ambiguities, such that a final selected wind solution is produced in every WVC.

4) Quality Control (QC): The primary objective of $\mathrm{QC}$ is to detect and reject poor-quality retrieved winds. Following the recent developments in the scatterometer data processing [23], [24], the CFOSCAT L2 processing will provide an estimation of the retrieved wind errors and rain probabilities. These two parameters will be defined as a function of the scatterometer quality indicator (typically the inversion MLE value) according to a postlaunch analysis of the collocated CFOSCAT and ancillary data.

\section{CFOSCAT SimUlATOR}

A prelaunch simulator has been developed to evaluate the CFOSCAT backscatter precision and retrieved wind quality under rain-free conditions. The overall simulation flow consists of two essential parts, i.e., the L1B simulator and the L2 processor, as shown in Fig. 3. The former is a radar simulator developed on the basis of the defined instrument parameters, and is used to assess the precision of the CFOSCAT backscatter measurements. This simulator consists of two relevant components, namely, the orbit propagator and the signal (X-factor) simulator. The orbit propagator is adapted from the Simplified Perturbations Models SGP4 [25], which is used to calculate the CFOSCAT telemetry and observing geometries. The $\mathrm{X}$-factor simulator accounts for the onboard signal processing and all the variables in the radar equation. Its primary objective is to estimate the signal-to-noise (ratio) and to derive the spatial response function (SRF) [8] for each measured slice, which are fundamental parameters for the evaluation of the instrument noise and resolution. Given a specific wind field and geophysical noise characteristic, which latter is the noise caused by the physical interaction of the radar signal and the scattering media, the backscatter coefficient $\sigma_{g}^{0}$ ("true" $\sigma^{0}$ perturbed by geophysical noise) for each measurement can be computed using the so-called NSCAT-4 Ku-band geophysical model function (GMF) [26] and the corresponding viewing geometries. Consequently, the simulated L1B product is generated after adding the instrument-induced random noise to the backscatter values $\sigma_{g}^{0}$. Note that the 10 -m-height surface 
winds from the European Centre for Medium-Range Weather Forecasts (ECMWF) are used as reference or "true" winds in this paper.

The L2 processor is actually adapted from the Pencilbeam scatterometer Wind Processor (PenWP) developed by the KNMI scatterometer team in the context of European Organisation for the Exploitation of Meteorological Satellites (EUMETSAT) Numerical Weather Prediction (NWP) Satellite Application Facility (SAF). The following simplifications/assumptions are used in this simulation.

1) The World Geodetic System 1984 is used as the reference coordinate system, and the topography effects are ignored when computing the $\mathrm{X}$ factors. Note that for land and ice applications, topography effects, which will affect the $\mathrm{X}$-factor computation, should be considered in the L1 processing.

2) The onboard processing is idealized in the simulation, i.e., the window effects, digital filter rolloff, and transmitter ramp/down are not taken into account by the simulator. Note though that the prelaunch test carried by the National Space Science Center shows that the overall effect of the abovementioned effects on the $\sigma^{0}$ precision is only about $0.02 \mathrm{~dB}$.

3) The simulated $\sigma^{0}$ values are well calibrated, such that the subtraction of thermal noise and the internal and external calibrations are not taken into account in the L1B simulator.

4) Only rain-free cases are considered, such that the geophysical noise is mainly induced by sea surface wind variability [23], [27]. Moreover, the L2 processor wind QC is omitted.

5) Since the real NWP forecast winds are used as "true" winds, the background winds used in the 2DVAR are actually simulated by adding noise to the "true" input winds. In principle, this noise is of similar characteristics to the geophysical noise used in the L1B simulator, i.e., they are both Gaussian noise but with different standard deviation (SD) errors. In this paper, various geophysical noise values are analyzed (see Section III-A), while the noise SD value used to derive the background winds is set to $1.5 \mathrm{~m} / \mathrm{s}$, which is a typical SD value according to [28].

More details about the CFOSCAT simulation are introduced in Sections III-A and III-B.

\section{A. $\sigma^{0}$ Simulation}

The measured $\sigma^{0}$ value basically contains two types of noise, i.e., the geophysical noise and the instrument noise. From the simulation point of view, the geophysical noise at WVC level depends on both the sub-WVC wind variability and the square root of the number of independent $\sigma^{0}$ samples [27]. In practice, since the sampling number and the wind variability have a similar effect on the geophysical noise (but with opposite trends), the former is set to a fixed approximate value, and the latter is derived from the Monte Carlo simulation that results in a simulated $\sigma^{0}$ distribution similar to that of the real measurements. Since no real CFOSCAT data are available now, we simply assume that the number of independent samples is the same as the number of slices within a certain WVC, and then simulate the geophysical noise with several different wind variability values. A more realistic assessment on the geophysical noise with real data is needed during the postlaunch campaign.

The "true" sea surface $\sigma^{0}$ value in Fig. 3 is derived from

$$
\sigma_{t}^{0}=f\left(\theta, p, w_{t}, \phi_{t}\right)
$$

where $f$ stands for the NSCAT-4 GMF, $\theta$ is the slice incidence angle, $p$ is the beam polarization, and $w_{t}$ and $\phi_{t}$ are the "true" wind speed and relative azimuth angle (i.e., relative angle between the beam azimuth and the wind direction). As aforementioned, to simulate noisy backscatter measurements due to geophysical noise, i.e., wind variability, the wind zonal $(u)$, and meridional $(v)$ wind components are depicted as follows:

$$
\left\{\begin{array}{l}
u_{g}=u_{t}\left(1+\varepsilon_{u} \cdot r_{u}\right) \\
v_{g}=v_{t}\left(1+\varepsilon_{v} \cdot r_{v}\right)
\end{array}\right.
$$

where $\left(u_{t}, v_{t}\right)$ represents the "true" wind vector simulated by using the ECMWF spatially and temporally interpolated to the slice acquisition location and time, $\varepsilon_{u}$ and $\varepsilon_{v}$ are the associated wind $u$ and $v$ component SD variability, and $r_{u}$ and $r_{v}$ are the random values generated from a normalized Gaussian distribution. Consequently, the $\sigma^{0}$ value including the effect of geophysical noise is written as

$$
\sigma_{g}^{0}=f\left(\theta, p, w_{g}, \phi_{g}\right)
$$

where $w_{g}$ and $\phi_{g}$ are converted from (5) by taking the beam azimuth angle information in account. In this paper, $\varepsilon_{u}$ and $\varepsilon_{v}$ are assumed to be identical, but several different $\varepsilon$ values (e.g., $0,0.5$, and $1 \mathrm{~m} / \mathrm{s}$ ) are used in the simulation to assess the CFOSCAT performances under different geophysical noise conditions. For a constant nonzero wind variability, the simulated geophysical noise is indeed speed dependent, i.e., it increases with decreasing wind speed, notably for low winds [27].

The measured $\sigma^{0}$ value is then simulated by adding the instrument noise to $\sigma_{g}^{0}$, i.e.,

$$
\sigma_{m}^{0}=\sigma_{g}^{0}\left(1+K_{p c} \cdot r\right)
$$

where $r$ is a set of random values generated from a normalized Gaussian distribution and $K_{p c}$ is the instrument-noise-induced normalized SD of the measurements. The derivation of $K_{p c}$ is presented in Section III-B.

\section{B. X-Factor Simulator}

The X-factor simulator is primarily used to calculate the $X$ values of the simulated 40 slices according to (3). Fig. 4(a) illustrates the $\mathrm{X}$ factor of the $\mathrm{VV}$ beam versus FFT bin indices (before aggregating to slices) at various azimuth angles. It shows that the bandwidth and the amplitude of the received signal vary with the antenna azimuth due to the Doppler shift effect. After integrating the FFT bins into a series of $10-\mathrm{km}$ slices, the integrated X shape of either the VV or the $\mathrm{HH}$ beam becomes independent of the antenna azimuth. In other words, the slice $\mathrm{X}$ values can be simply depicted 

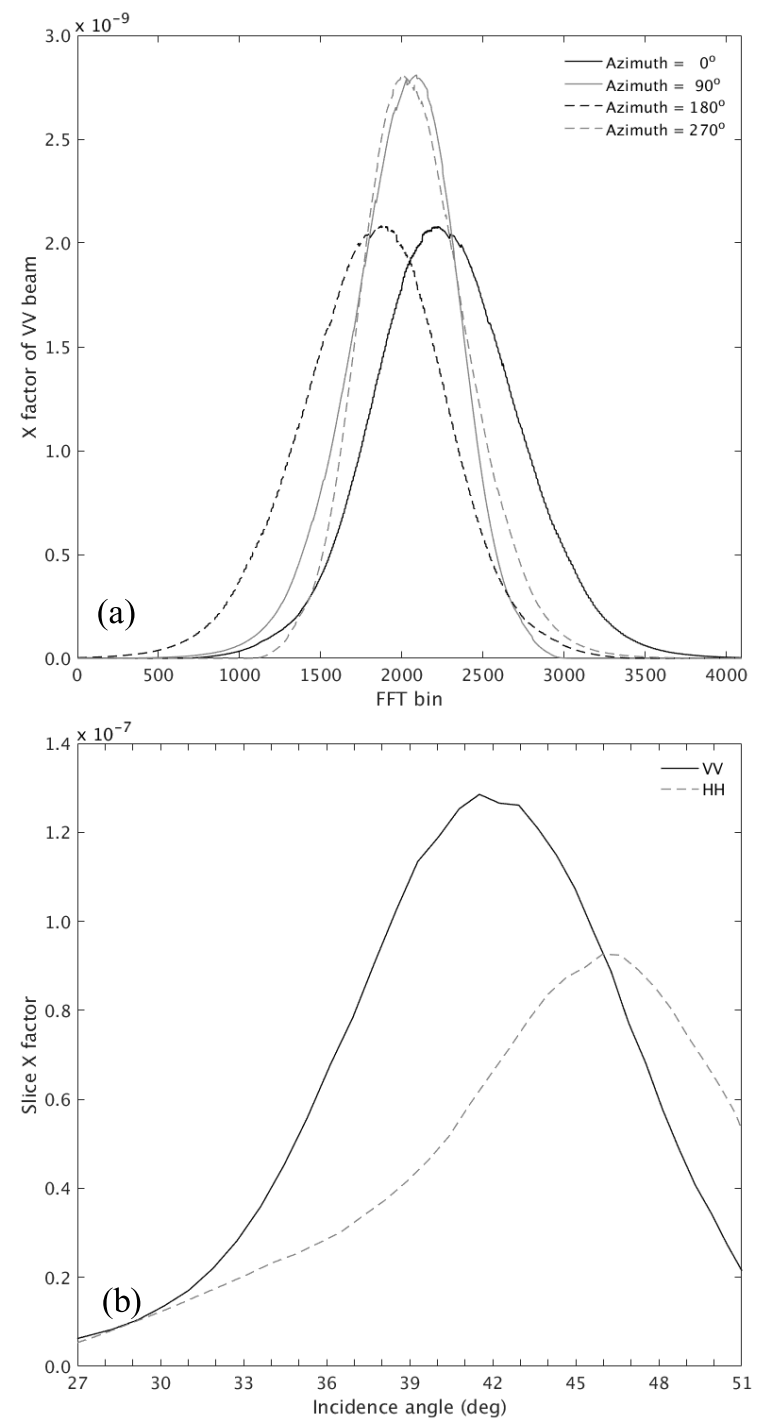

Fig. 4. (a) $\mathrm{X}$ values versus FFT bin indices at various antenna azimuth angles (see legend) for $\mathrm{VV}$ beam. (b) $\mathrm{X}$ values of the simulated 40 slices as a function of incidence angle for VV (solid curve) and $\mathrm{HH}$ (dashed curve) beams.

as a function of incidence angle, as shown in Fig. 4(b). Note that the satellite altitude is not fixed, so the slice $\mathrm{X}$ value at a given incidence angle actually changes with the satellite altitude, and thus with latitude. Consequently, it is proposed to construct a 2-D X LUT (e.g., satellite latitude and slice index) in order to reduce the required computing resources in both the simulation and the ground processing.

The X-factor simulator is also used to derive the SRF of CFOSCAT, which is a 2-D function representing the characteristics of the surface slice cells. Physically, the SRF is constructed from (3) by taking the energy contribution to the slice from each scattering patch, and then normalizing by the patch area, i.e.,

$$
\operatorname{SRF}_{i}(\vec{v})=\frac{P_{t} G_{p}^{2} G_{r} \lambda^{2}}{(4 \pi)^{3} L} \frac{g_{j}^{2}}{r_{j}^{4}} \sum_{k=k_{s}}^{k_{e}}\left\{\frac{\sin \left[\pi N_{j}\left(f_{b, j} T-\frac{k}{N}\right)\right]}{\sin \left[\pi\left(f_{b, j} T-\frac{k}{N}\right)\right]}\right\}^{2}
$$

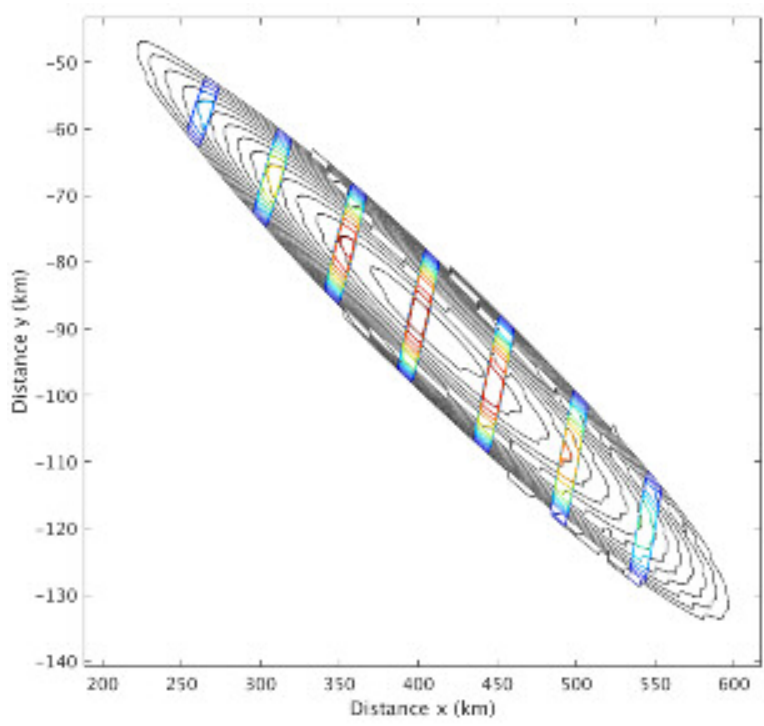

Fig. 5. SRF for the entire CFOSCAT footprint (black contours) and SRFs for several sample slices (color contours) of width $\sim 10 \mathrm{~km}$. The illustration corresponds to a simulated VV beam with an azimuth angle of $270^{\circ}$ (as defined in Section II-A). A contour spacing of $1-\mathrm{dB}$ interval is used.

where $\vec{v}$ is the surface position vector. Fig. 5 illustrates the SRF for the whole CFOSCAT footprint (black contours) and the SRFs for several sample slices (color contours) of width $\sim 10 \mathrm{~km}$. To display the slice SRFs clearly, the distance between two consecutive sample slices in Fig. 5 is about $50 \mathrm{~km}$. The measured radar cross section is an average of the surface $\sigma^{0}$ weighted by the SRF. For a beam azimuth angle of $90^{\circ}$ or $270^{\circ}$ (i.e., side looking; see the definition in Section II-A), there is a slight tilt in the orientation of the slice due to the Doppler shift across the footprint. This should be taken into account when developing a high-resolution wind product over the coastal areas [29].

Due to the radar fading and the presence of thermal noise, the backscatter measurements are noisy. The measurement precision is commonly quantified by the parameter $K_{p c}$, which, in the case of range filtering, is expressed as [8]

$$
K_{p c}=\sqrt{A+\frac{B}{\mathrm{SNR}}+\frac{C}{\mathrm{SNR}^{2}}}
$$

where coefficients $A, B$, and $C$ are approximated to

$$
A=\frac{1}{B_{s} \tau_{p}} \quad B=\frac{2}{B_{s} \tau_{g}} \quad C=\frac{1}{B_{s} \tau_{g}}
$$

where $B_{s}$ is the slice bandwidth, $\tau_{p}$ is the pulse duration, and $\tau_{g}$ is the length of the receiving window $(\sim 2.7 \mathrm{~ms})$. Note that (10) is a simplified expression from the analytical expression in [8]. The difference between parameters $A$, $B$, and $C$ derived from (10) and those from the analytical expression in [8] is generally smaller than 3\% [18]. As such, (10) is implemented in both the simulator and the operational $\mathrm{L} 1$ processor. Finally, the measurement SNR is given by

$$
\mathrm{SNR}=\frac{X \sigma_{t}^{0}}{B_{s} \tau_{g} n_{0}}
$$

where $n_{0}$ is the noise power spectra density. 

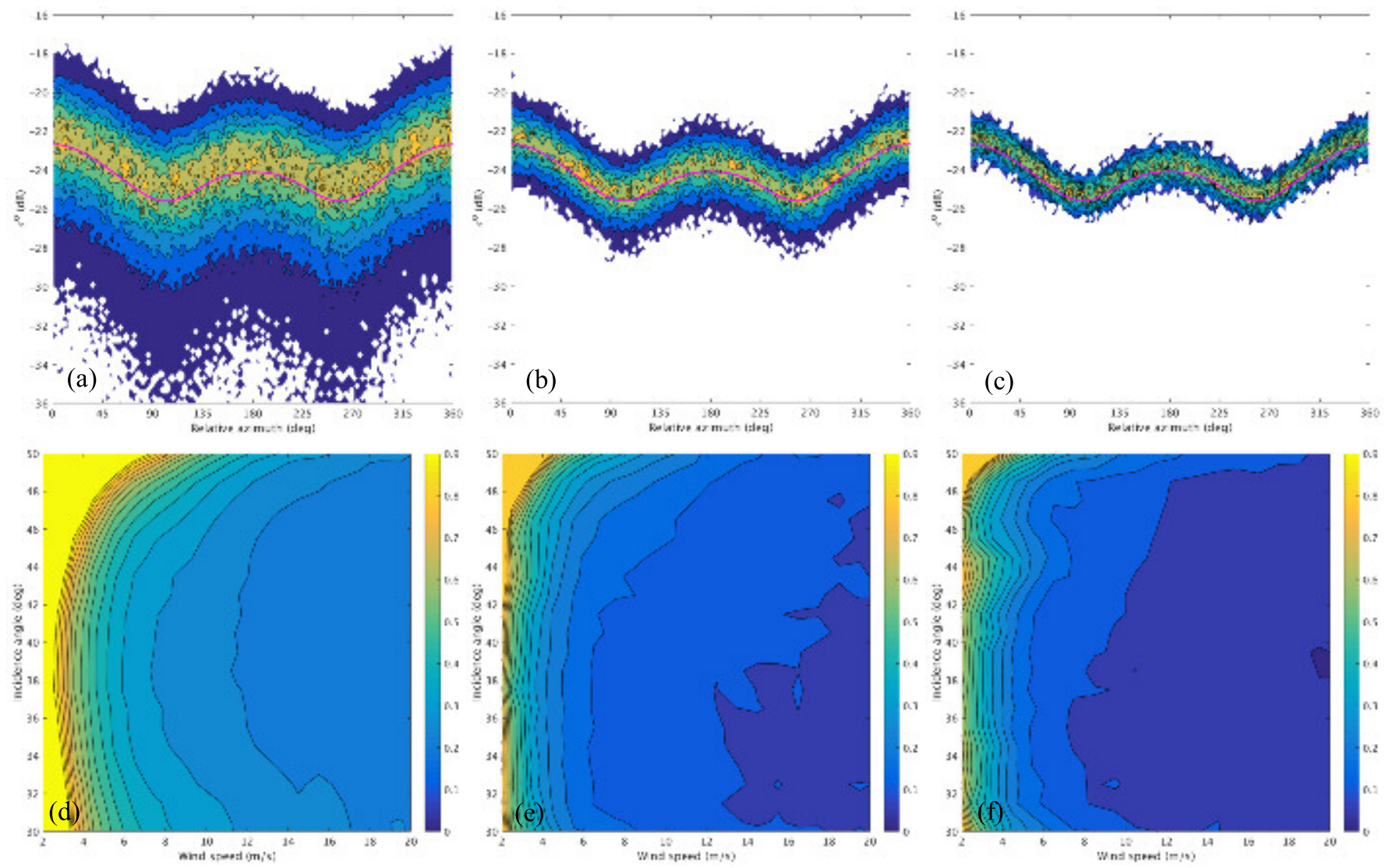

Fig. 6. 2-D histogram of the simulated $\sigma^{0}$ (VV beam) versus the relative azimuth angle $\phi_{g}$ for $\theta=40^{\circ}$ and $w_{t}=4 \mathrm{~m} / \mathrm{s}$, and for a (a) slice $\sigma^{0}$, (b) WVC-mean $\sigma^{0}$ with a grid resolution of $12.5 \mathrm{~km}$, and (c) WVC-mean $\sigma^{0}$ with a grid resolution of $25 \mathrm{~km}$. The mangeta curves represent the "true" $\sigma^{0}$ values derived from the NSCAT-4 GMF. Contour plots of the normalized measurement error $\left(K_{p}, \mathrm{VV}\right.$ beam $)$ as a function of wind speed $(x$-axis $)$ and incidence angle ( $y$-axis), and for a (d) slice $K_{p}$, (e) WVC-mean $K_{p}$ with a grid resolution of $12.5 \mathrm{~km}$, and (f) WVC-mean $K_{p}$ with a grid resolution of $25 \mathrm{~km}$. Note that a contour spacing of 0.05 is used.

\section{EXPECTED PERformances}

\section{A. Backscatter Precision}

In order to evaluate the overall backscatter precision of CFOSCAT, 10 days of L1B data ( $\sim 150$ orbits) are simulated using the aforementioned L1B simulator. The overall backscatter precision is depicted by parameter $K_{p}$ as follows:

$$
K_{p}=\frac{\sqrt{\operatorname{var}\left(\sigma^{0}\right)}}{E\left(\sigma^{0}\right)}
$$

where $E\left(\sigma^{0}\right)$ is the mean backscatter and $\operatorname{var}\left(\sigma^{0}\right)$ is its associated variance. It consists of the contributions from both the instrument noise (denoted by $K_{p c}$ ) and the geophysical noise. Various wind variability values (e.g., $\varepsilon=0,0.5$, and $1 \mathrm{~m} / \mathrm{s}$ ) are considered in the geophysical noise simulation. For example, the top plots of Fig. 6 illustrate the 2-D histograms of the simulated $\sigma^{0}$ values for (a) $10-\mathrm{km}$ slices, (b) $12.5-\mathrm{km}$ WVCs, and (c) $25-\mathrm{km}$ WVCs observed by the VV beam at $\theta=40^{\circ}$ and $w_{t}=4 \mathrm{~m} / \mathrm{s}$, with an $\varepsilon$ of $0.5 \mathrm{~m} / \mathrm{s}$. It is clear that the measurement uncertainty is reduced remarkably after aggregating slices into WVC. The measurement uncertainty decreases with increasing WVC size, as the number of slices being averaged increases. The bottom plots of Fig. 6 present the expected measurement precision as a function of wind speed and incidence angle for the corresponding top plot cases. It shows that the $K_{p}$ value increases rapidly as the wind speed decreases, particularly for $w_{t}$ below $4 \mathrm{~m} / \mathrm{s}$. Moreover, the $K_{p}$ value is modulated by the incidence angle, which is consistent with the $\mathrm{X}$ shape shown in Fig. 4(b). The slice $K_{p}$ value is generally high (>20\%). Nonetheless, the WVC $K_{p}$ is very much reduced after aggregating slices with similar incidence and azimuth angles into a specific WVC view. For the WVC-mean $\sigma^{0}$ s with $\theta<45^{\circ}$, the 12.5 -km WVC-mean $K_{p}$ is lower than $20 \%$ for $w_{t}>5 \mathrm{~m} / \mathrm{s}$, and lower than $10 \%$ for $w_{t}>10 \mathrm{~m} / \mathrm{s}$. This value is further reduced by at least $2 \%$ for the 25-km WVC. More interestingly, the $K_{p}$ dependence on incidence angle is reduced after aggregating slices to WVC.

According to Fig. 4(b), the $\mathrm{X}$ value of the $\mathrm{HH}$ beam is lower than that of the VV beam for $\theta<46^{\circ}$, such that the measurement SNR of the former is generally lower than that of the latter, which in turn results in higher $K_{p}$ value. At high incidence angles $\left(\theta>46^{\circ}\right)$, although the $\mathrm{X}$ value of the $\mathrm{HH}$ beam is higher than that of the VV beam, it does not lead to remarkably lower $K_{p}$ values, because the backscatter value of the HH beam is lower than that of the VV beam (not shown).

The impact of the wind-variability-induced geophysical noise on the measurement precision is also analyzed. As expected, the slice $K_{p}$ value generally increases with wind 
variability $\varepsilon$ (not shown). Moreover, the degradation of the measurement precision due to geophysical noise is more striking for wind speeds between 4 and $5 \mathrm{~m} / \mathrm{s}$ than for other wind speed intervals. For the most sensitive wind speed interval, the slice $K_{p}$ value is increased by $5 \%(15 \%)$ when increasing the wind variability from 0 to $0.5 \mathrm{~m} / \mathrm{s}(1 \mathrm{~m} / \mathrm{s})$. At low winds, the measurement SNR is generally low for Ku-band scatterometers, such that the instrument noise $K_{p c}$ is high, and in turn dominates the measurement precision. However, at high winds, the ratio of wind variability to wind speed is low. As such, the impact of wind variability on the total $K_{p}$ decreases with increasing wind speeds.

At the WVC level, however, the $K_{p}$ value does not significantly increase $(<1 \%)$ with increasing wind variability. This is probably due to the fact that many slices (20-30) are averaged into a specific WVC view, and the geophysical noise is assumed to be uncorrelated among those slices. This assumption should be further verified in the postlaunch campaign.

\section{B. Wind Inversion Skill}

The MLE used to invert the CFOSCAT winds is defined as

$$
\text { MLE }=\frac{1}{N} \sum_{i}^{N} \frac{\left(\sigma_{m i}^{0}-\sigma_{s i}^{0}\right)^{2}}{\left(K_{p i} \cdot \sigma_{m i}^{0}\right)^{2}}
$$

where $N$ is the number of measurements at different azimuth angles (or views), $\sigma_{m i}^{0}$ is the backscatter measured by the $i$ th beam, $\sigma_{s i}^{0}$ is the backscatter simulated through the NSCAT-4 GMF, and $K_{p i}$ is the normalized SD of the backscatter measurements. The wind solutions corresponding to the minimum MLE values (typically up to 4) are used for AR purposes.

Fig. 7(a) illustrates the minimum MLE value as a function of wind direction for several WVCs with different distances to the subsatellite track. Fig. 7(b) shows the corresponding wind solutions. A wind variability $\varepsilon$ of $0.5 \mathrm{~m} / \mathrm{s}$ and a "true" wind speed and direction of about $4.5 \mathrm{~m} / \mathrm{s}$ and $300^{\circ}$, respectively, are used in the simulation. Similar to the pencil-beam scatterometers, the wind retrieval skill of CFOSCAT depends very much on the WVC position across the swath. For the outerswath regions (WVC distance to sub-satellite track $\sim 500 \mathrm{~km}$ ), all the views are acquired at similar incidence and azimuth angles (i.e., poor incidence and azimuth diversity), resulting in a large wind ambiguity (i.e., very broad local minima). For WVCs with a distance to subsatellite track of $\sim(150,400) \mathrm{km}$, the measurements have good incidence and azimuth diversity, such that the cost function has well-defined minima, leading to a small wind ambiguity. Although the azimuth diversity is poor in the nadir region (i.e., close to the subsatellite track), the inversion skill is slightly better than that of the outer regions, because the former has more incidence angle diversity. In practice, however, a set of backscatters at different incidence angles should be well calibrated, or else it may lead to biased wind results and increased wind errors.

\section{Example}

A typical meteorological case is examined in this section in order to better illustrate the potential performance
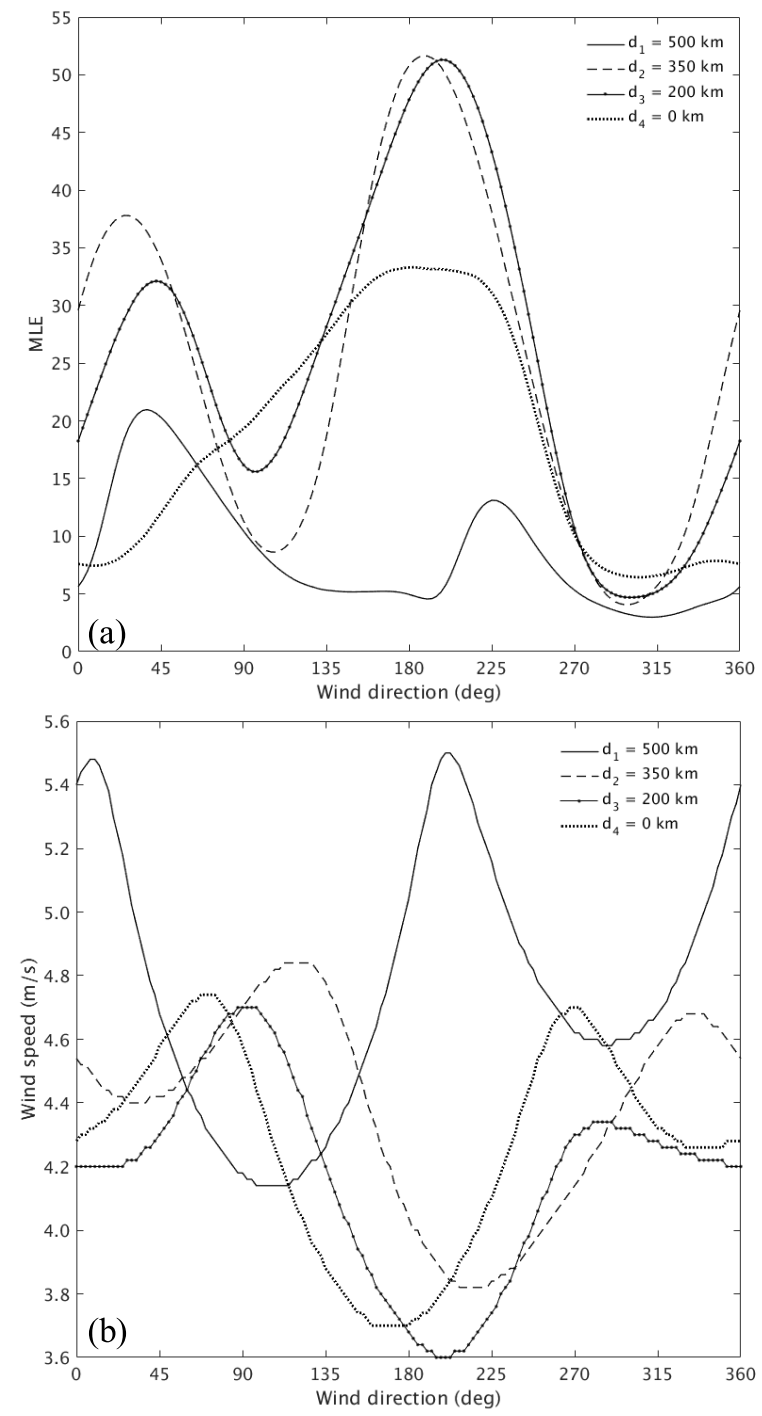

Fig. 7. (a) Minimum MLE value as a function of wind direction for several WVCs with different distances to the subsatellite track. (b) Corresponding wind solution versus wind direction. Here, the "true" wind speed and direction are about $4.5 \mathrm{~m} / \mathrm{s}$ and $300^{\circ}$, respectively.

of CFOSCAT. Again, the wind variability is assumed to be $0.5 \mathrm{~m} / \mathrm{s}$ in the simulation. Moreover, since the L1B simulator does not account for rain effects, QC is not performed. Fig. 8(a) shows a simulated CFOSCAT retrieved wind field with a grid resolution of $25 \mathrm{~km}$, using the standard MLE inversion output (up to four ambiguous wind solutions) in combination with the 2DVAR. In the nadir region, i.e., the center line along the swath, it is clear that the retrieved wind field is spatially inconsistent compared to the "true" wind field (not shown). This is due to the fact that only the MLE minima are accounted for, while the presence of broad minima [see dotted curve in Fig. 7(a)] suggests that the neighboring wind solutions (with similar MLE values, and therefore similar probability of being the true wind) should also be considered [28]. Fig. 8(b) shows the same wind field as in Fig. 8(a), but retrieved using the multiple solution scheme (MSS, i.e., up to 144 ambiguous wind solutions) [30] in combination with the 2DVAR. Since MSS provides solutions in the direction of the 


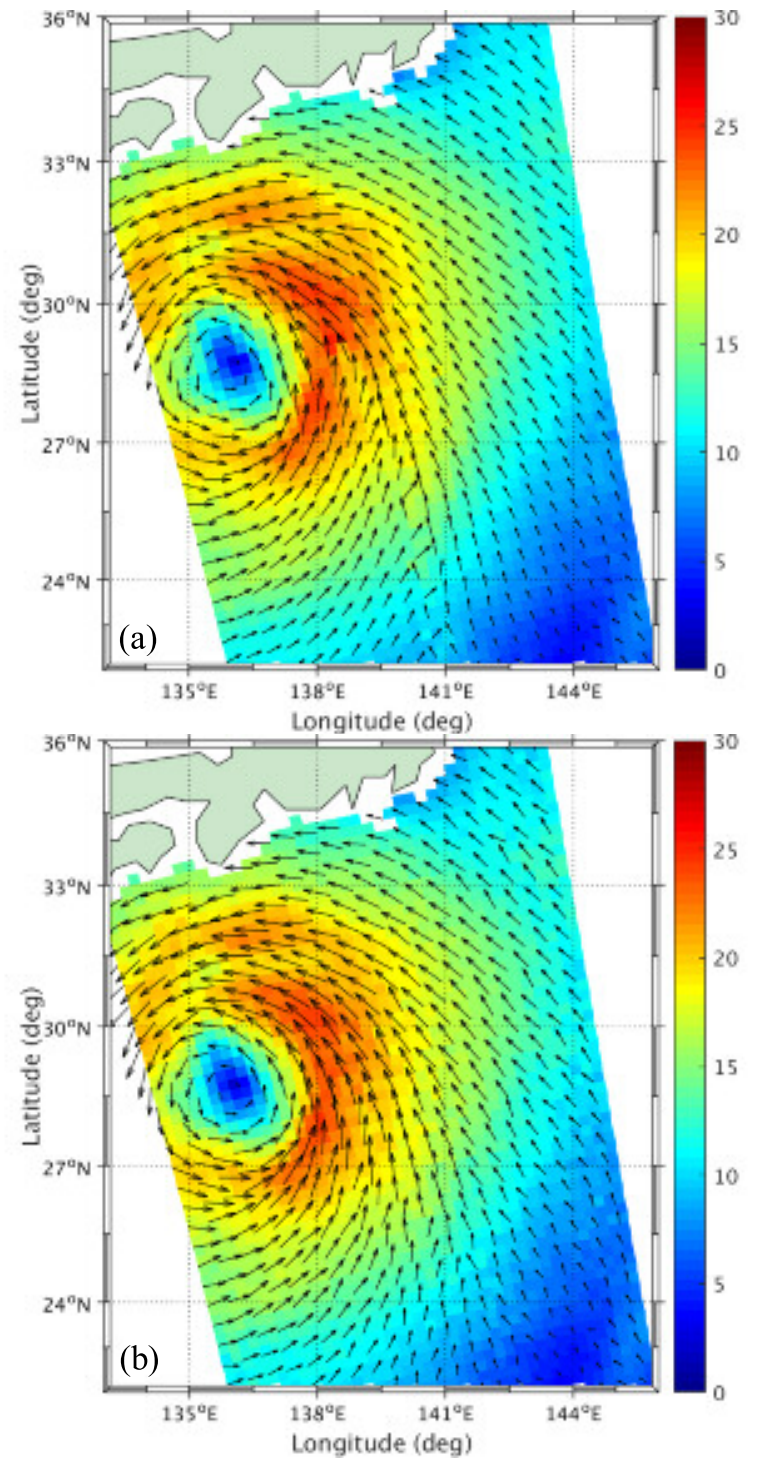

Fig. 8. (a) Simulated CFOSCAT retrieved wind field using the standard MLE output with up to four ambiguities and the 2DVAR AR. (b) Simulated MSS retrieved wind field using the standard MLE output with up to four ambiguities and the 2DVAR AR.. The colorbar indicates the retrieved wind speed.

mean flow over the nadir regions, a more spatially consistent wind field is achieved after AR. A few inconsistent wind arrows persist in Fig. 8(b), which should be quality controlled in the real data processing.

The performance of the CFOSCAT wind retrieval at $12.5-\mathrm{km}$ grid resolution is also analyzed. In general, the retrieved $12.5-\mathrm{km}$ wind field is similar to that of Fig. 8, although winds at higher grid resolution are a little nosier than those at lower resolution (not shown), particularly under low wind conditions. This is because a smaller number of slices is averaged into higher resolution WVC, leading to larger measurement noise. Fig. 9 illustrates the MLE value of the retrieved wind field at $25-$ and $12.5-\mathrm{km}$ resolution, respectively. In the vicinity of the low-pressure center, the area of large MLE values is larger at the lower grid resolution [Fig. 9(a)]. This is due to the fact that when aggregating slices into an averaged $\sigma^{0}$ over dynamic (variable) areas,

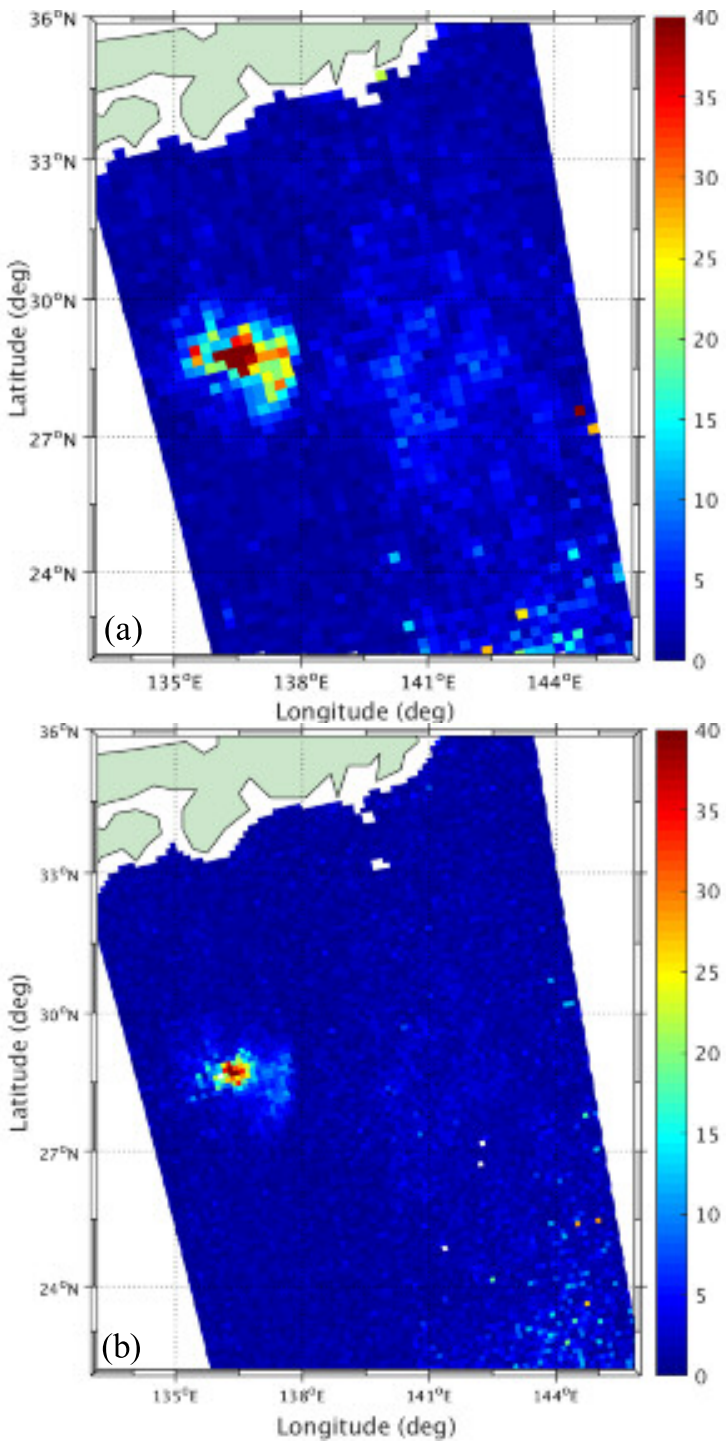

Fig. 9. MLE value (see the colorbar) of the retrieved wind field in Fig. 8(b), at (a) $25-\mathrm{km}$ grid resolution and (b) $12.5-\mathrm{km}$ grid resolution.

the larger the area, the less representative this $\sigma^{0}$ is of a mean "uniform" WVC wind. Fig. 9(b) shows a few WVCs without retrieved wind information (see the white blocks in the swath). This is because the measurements at $12.5-\mathrm{km}$ resolution are generally noisier than those at $25-\mathrm{km}$ resolution, such that the inversion is more likely to encounter a cost function without well-defined local minima, i.e., case without wind solution. Nonetheless, Fig. 9 implies that the higher resolution product can be used to better identify the sharp transition in the wind field, which is relevant for certain applications, such as nowcasting and disaster monitoring.

\section{Retrieved Wind Quality}

Following the analysis in Section IV-C, an overall assessment on the performance of the CFOSCAT wind retrieval is carried out using the MSS inversion scheme in combination with the 2DVAR, and globally realistic wind speed distributions. Table II summarizes the retrieved CFOSCAT 
TABLE II

EXPECTED CFOSCAT WIND QUALITY AT DIFFERENT GRID RESOLUTIONS AND GEOPHYSICAL NOISE CONDITIONS. THE SD VALUes of THE DifFerence BeTWEen RETRIEVED WINDS AND INPUT ("TRUE") ECMWF WINDS ARE ESTIMATED

\begin{tabular}{|c|c|c|c|c|c|}
\hline \multirow[b]{2}{*}{ Resolution } & \multirow{2}{*}{$\begin{array}{l}\text { Geophysical } \\
\text { noise }(\mathrm{m} / \mathrm{s})\end{array}$} & \multicolumn{4}{|c|}{ SD values of wind } \\
\hline & & $\begin{array}{l}\text { speed } \\
(\mathrm{m} / \mathrm{s})\end{array}$ & $\begin{array}{l}\text { dir } \\
\left(^{\circ}\right)\end{array}$ & $\begin{array}{l}u \\
(\mathrm{~m} / \mathrm{s})\end{array}$ & $\begin{array}{l}v \\
(\mathrm{~m} / \mathrm{s})\end{array}$ \\
\hline \multirow{3}{*}{$25 \mathrm{~km}$} & 0.0 & 0.34 & 13.6 & 1.15 & 1.21 \\
\hline & 0.5 & 0.35 & 13.6 & 1.14 & 1.22 \\
\hline & 1.0 & 0.37 & 13.6 & 1.14 & 1.22 \\
\hline \multirow{3}{*}{ 12. $5 \mathrm{~km}$} & 0.0 & 0.43 & 15.1 & 1.23 & 1.33 \\
\hline & 0.5 & 0.43 & 15.2 & 1.24 & 1.34 \\
\hline & 1.0 & 0.45 & 15.3 & 1.25 & 1.34 \\
\hline
\end{tabular}

wind quality (SD errors) at various grid resolutions and wind variability conditions. Similar to the verification of other scatterometers, the statistics of wind direction are computed for winds above $4 \mathrm{~m} / \mathrm{s}$. Since the simulated $\sigma^{0}$ values are assumed to be well calibrated, the biases of wind speed, direction, $u$ and $v$ components are all close to zero (not shown). As expected, the wind quality of the $12.5-\mathrm{km}$ WVCs is a little worse than that of the $25-\mathrm{km}$ WVCs. Moreover, the statistical scores slightly degrade with increasing geophysical noise, for both 25- and 12.5-km WVCs.

Given the wind variability of $0.5 \mathrm{~m} / \mathrm{s}$, Fig. 10 shows the vector root-mean-square (VRMS) difference between the retrieved and the "true" winds as a function of cross-track position [Fig. 10(a)] and the average retrieved/"true" wind speed [Fig. 10(b)]. It shows that the error statistics degrade rapidly for WVCs at the nadir region or at low wind conditions. Although the wind inversion skill in the nadir region is slightly better than that of the outer regions [Fig. 7(a)], the retrieved wind quality of the outer WVCs is similar (12.5-km grids) or even better (25-km grids) than that of the nadir WVCs. This implies that AR has more remarkable impact on the outer regions than the nadir WVCs.

\section{E. AR Sensitivity to Background Winds}

In above simulation experiments, the background winds used in 2DVAR are simulated by adding a noise SD value of $1.5 \mathrm{~m} / \mathrm{s}$ to the "true" input winds. Using another background wind field with lower (higher) error SD may lead to better (worse) verification scores than in Table II. For instance, if the "true" winds are used as 2DVAR background winds (note that this ideal case never happens in the real data processing), the SD errors of retrieved $25-\mathrm{km}$ wind direction and $u / v$ components are remarkably reduced to $5^{\circ}$ and $\sim 0.5 \mathrm{~m} / \mathrm{s}$, which are better than the quality scores of convention pencil-beam scatterometers [28]. Nonetheless, the SD errors of retrieved wind speed are the same as those in Table II.

From the simulation point of view, adding noise to the "true" winds gives the background fields random high-spatialfrequency errors that are unlikely to exist in actual NWP background fields. A better approach might be to use ECWMF
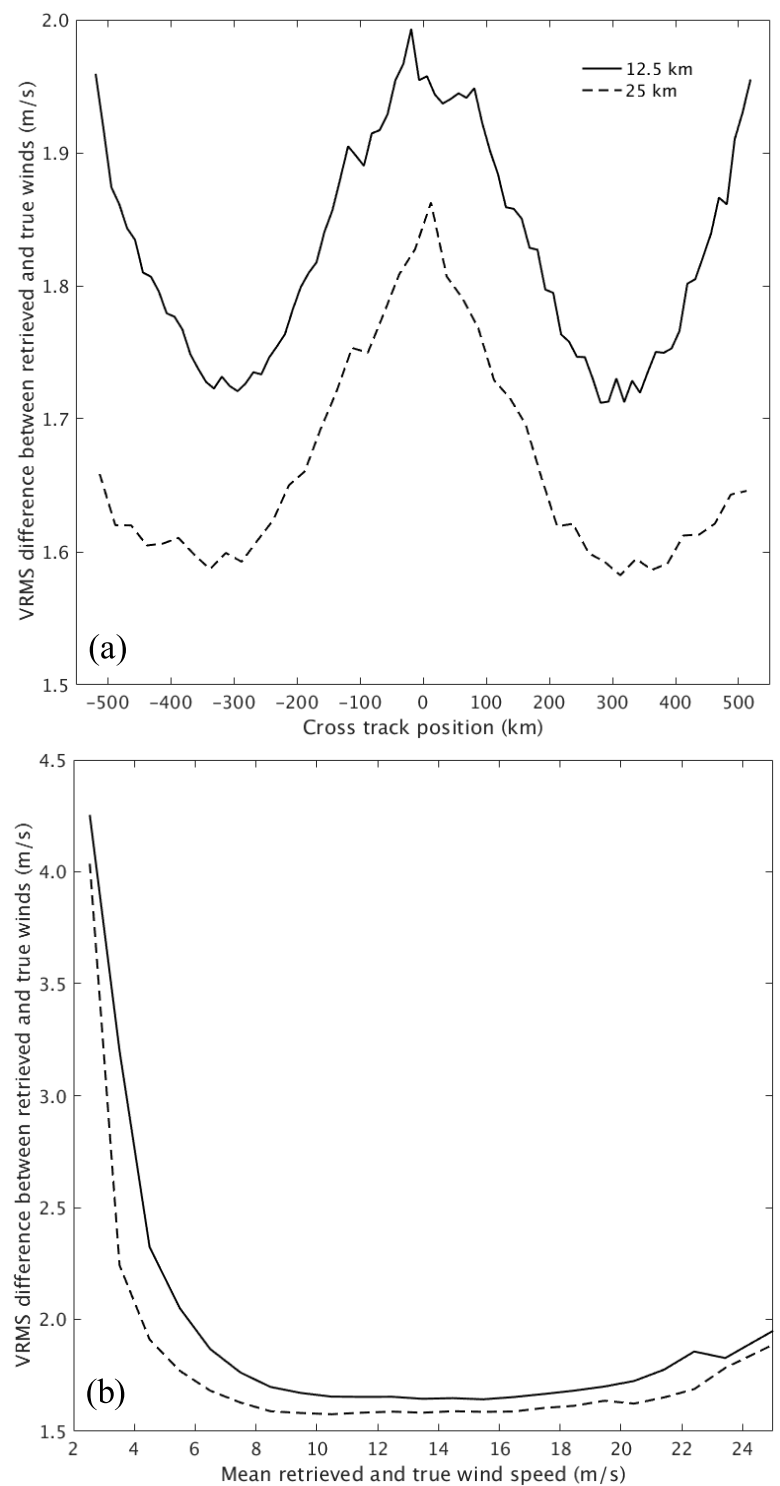

Fig. 10. VRMS difference between the retrieved and the "true" winds as a (a) function of (a) cross-track position and (b) mean retrieved/"true" wind speed. Solid curve for the $12.5-\mathrm{km}$ grid resolution, and dashed curve for the $25-\mathrm{km}$ grid resolution.

fields that are slightly misplaced in time from the "true" field as background wind fields. For instance, the background wind fields which are $6 \mathrm{~h}$ before the CFOSCAT acquisitions correspond to an SD error of $1.3 \mathrm{~m} / \mathrm{s}$ for the $u$ component and of $1.8 \mathrm{~m} / \mathrm{s}$ for the $v$ component, comparing to the "true" field. These SD errors are close to the typical background errors of $1.5 \mathrm{~m} / \mathrm{s}$ [28]. Consequently, an extra experiment using the misplaced ECMWF background fields (i.e., $6 \mathrm{~h}$ before the CFOSCAT measurements) for the AR is tested, in which case, the overall SD errors of retrieved $25-\mathrm{km}$ wind direction and $u / v$ components are $7.7^{\circ}, 0.67 \mathrm{~m} / \mathrm{s}$, and $0.71 \mathrm{~m} / \mathrm{s}$, respectively, which are worse than the scores of the previous extreme case (using "true" wind fields as background) but better than the scores of the simulations in Section IV-D. This implies that the current AR is very sensitive to the background winds. Consequently, the configurations of MSS and 2DVAR need to be further refined at the postlaunch stage. 


\section{CONCLUSion}

The rotating fan-beam scatterometer designed and developed for the future CFOSAT mission is a new type of spaceborne scatterometer. This instrument is a real aperture, conical scanning fan-beam radar, which combines the characteristics of the fixed fan-beam scatterometer and the rotating pencilbeam scatterometer, and provides unprecedented observation geometries for the retrieval of sea surface wind vector fields. The system design and essential parameters of CFOSCAT are summarized in this paper. Furthermore, a thorough simulation is carried out to evaluate the precision of its backscatter measurements and the quality of the retrieved wind vectors.

The normalized measurement error $\left(K_{p}\right)$ of the CFOSCAT $10-\mathrm{km}$ slices is generally above $20 \%$, and it increases with decreasing wind speed. By aggregating slices with similar incidence and azimuth angles into a specific WVC view, the $K_{p}$ value of the WVC-mean $\sigma^{0}$ s is reduced to $5 \%-20 \%$, depending on the surface wind speed conditions. The impact of the geophysical noise on the slice measurement precision is nonnegligible, particularly for wind speeds below $5 \mathrm{~m} / \mathrm{s}$. However, the geophysical noise does not substantially degrade the measurement precision of WVC $\sigma^{0} \mathrm{~s}$. This is because many slices are averaged to get a specific WVC $\sigma^{0}$ value, and the geophysical noise among those slices are assumed to be uncorrelated. A more realistic assessment of the geophysical noise impact on the backscatter precision should be carried out during the postlaunch campaign.

Provided that the backscatter measurements are under rainfree condition and well calibrated, the expected wind quality is fully compliant with the scientific requirements, i.e., a wind speed SD error $<2 \mathrm{~m} / \mathrm{s}$ and a wind direction SD error $<20^{\circ}$, as proposed by NSOAS. The wind direction skill, however, is sensitive to the WVC position across the swath. The use of MSS and 2DVAR can improve the wind retrieval quality remarkably, particularly for WVCs at satellite nadir region. The simulation also shows the wind direction skill of CFOSCAT is very sensitive to the background wind quality, suggesting that further developments of the L2 processing with MSS and 2DVAR are required at the postlaunch stage.

The innovative observation geometry of CFOSCAT is not fully exploited in this paper. This should be carefully investigated using real data, i.e., after the satellite launch. On the one hand, observations with different geometries (views) at the same WVC should be relatively calibrated to high accuracy of about $0.2 \mathrm{~dB}$, which is a difficult requirement to meet for the prelaunch calibration of scatterometer fan-beam antennas [31]. On the other hand, the rain impact on the backscatter measurements depends on incidence angle. Backscatter measurements at higher incidence angles correspond to longer range distances across the rain cell, and to larger rain splashing effect on the ocean surface (because of smaller Bragg wavelength [32]). Such incidence-dependent rain effect may be beneficial to the wind QC of the CFOSCAT L2 processing. More recently, it has been found that the sea surface temperature effects on the Ku-band radar backscatter are not negligible, which are incidence dependent and more pronounced in $\mathrm{VV}$ beam than in $\mathrm{HH}$ beam [33], and in turn, challenge the CFOSCAT wind retrieval. In summary, further efforts with real data analysis are needed to improve the CFOSCAT L1 and L2 processing.

\section{ACKNOWLEDGMENT}

The 2DVAR module used in this paper is adapted from the PenWP software which was developed through the EUMETSAT NWP SAF. The SGP4 routines are provided by $\mathrm{D}$. Vallado through his webpage http://www.smad. com/vallado/. The authors would like to thank all of their colleagues for their valuable contributions to the development of the CFOSAT RFSCAT instrument. They would also like to thank the three reviewers who helped to improve this paper.

\section{REFERENCES}

[1] J. Johnson, L. Williams, E. Bracalente, F. Beck, and W. Grantham, "Seasat-A satellite scatterometer instrument evaluation," IEEE J. Ocean. Eng., vol. OE-5, no. 2, pp. 138-144, Apr. 1980.

[2] W. Y. Tsai et al., "Postlaunch sensor verification and calibration of the NASA Scatterometer," IEEE Trans. Geosci. Remote Sens., vol. 37, no. 3, pp. 1517-1542, May 1999.

[3] F. M. Naderi, M. H. Freilich, and D. G. Long, "Spaceborne radar measurement of wind velocity over the ocean-An overview of the NSCAT scatterometer system," Proc. IEEE, vol. 79, no. 6, pp. 850-866, Jun. 1991.

[4] A. Stoffelen and D. L. T. Anderson, "Wind retrieval and ERS-1 scatterometer radar backscatter measurements," Adv. Space Res., vol. 13, no. 5 , pp. 53-60, 1993.

[5] J. Figa-Saldaña, J. J. W. Wilson, E. Attema, R. Gelsthorpe, M. R. Drinkwater, and A. Stoffelen, "The Advanced Scatterometer (ASCAT) on the meteorological operational (MetOp) platform: A follow on for European wind scatterometers," Can. J. Remote Sens., vol. 28, no. 3, pp. 404-412, 2002.

[6] A. Verhoef, M. Portabella, and A. Stoffelen, "High-resolution ASCAT scatterometer winds near the coast," IEEE Trans. Geosci. Remote Sens. vol. 50, no. 7, pp. 2481-2487, Jul. 2012.

[7] M. W. Spencer, C. Wu, and D. G. Long, "Tradeoffs in the design of a spaceborne scanning pencil beam scatterometer: Application to SeaWinds," IEEE Trans. Geosci. Remote Sens., vol. 35, no. 1, pp. 115-126, Jan. 1997.

[8] M. W. Spencer, C. Wu, and D. G. Long, "Improved resolution backscatter measurements with the SeaWinds pencil-beam scatterometer," IEEE Trans. Geosci. Remote Sens., vol. 38, no. 1, pp. 89-104, Jan. 2000.

[9] S. A. Bhowmick, R. Kumar, and A. S. K. Kumar, "Cross calibration of the OceanSAT-2 scatterometer with QuikSCAT scatterometer using natural terrestrial targets," IEEE Trans. Geosci. Remote Sens., vol. 52, no. 6, pp. 3393-3398, Jun. 2014

[10] Z. Wang, C. Zhao, J. Zou, X. Xie, Y. Zhang, and M. Lin, "An improved wind retrieval algorithm for the HY-2A scatterometer," Chin. J. Oceanol. Limnol., vol. 33, no. 5, pp. 1201-1209, 2015.

[11] N. M. Madsen and D. G. Long, "Calibration and validation of the RapidScat scatterometer using tropical rainforests," IEEE Trans. Geosci. Remote Sens., vol. 54, no. 5, pp. 2846-2854, May 2016.

[12] N. Ebuchi, "Evaluation of marine surface vectors observed by SCATSAT-1 scatterometer," presented at the Int. Ocean Vector Winds Sci. Team Meeting, San Diego, CA, USA, 2017. Accessed: Mar. 23, 2018. [Online]. Available: https://mdc.coaps.fsu.edu/ scatterometry/meeting/docs/2017/docs/Tuesday/morning/SecondSession/ 1045_Ebuchi_IOVWST2017_SCATSAT.pdf

[13] C.-C. Lin, B. Rommen, J. J. W. Wilson, F. Impagnatiello, and P. S. Park, "An analysis of a rotating, range-gated, fanbeam spaceborne scatterometer concept," IEEE Trans. Geosci. Remote Sens., vol. 38, no. 5, pp. 2114-2121, Sep. 2000.

[14] C.-C. Lin, A. Stoffelen, J. de Kloe, V. R. Wismann, S. Bartha, and H.-R. Schulte, "Wind retrieval capability of rotating, range-gated, fanbeam spaceborne scatterometer," Proc. SPIE, Sensors, Syst., Next-Gener. Satell. VI, vol. 4881, pp. 268-279, Apr. 2003.

[15] W. Lin and X. Dong, "Design and optimization of a Ku-band rotating, range-gated fanbeam scatterometer,' Int. J. Remote Sens., vol. 32, no. 8, pp. 2151-2171, 2011 
[16] W. Lin, X. Dong, and D. Zhu, "Performance simulation of a spaceborne dual-frequency Rotating Fanbeam SCATterometer," in Proc. IEEE Int. Geosci. Remote Sens. Symp. (IGARSS), Vancouver, BC, Canada, Jul. 2011, pp. 941-944.

[17] V. Y. Karaev et al., "Russian scatterometer: Discussion of the concept and the numerical simulation of wind field retrieval," Int. J. Remote Sens., vol. 36, no. 24, pp. 6056-6084, 2015.

[18] W. Lin, "Study on spaceborne rotating, range-gated, fanbeam scatterometer system," (in Chinese), Ph.D. dissertation, Graduate Univ. Chin Acad. Sci., Beijing, China, 2011.

[19] V. Wismann, A. Stoffelen, J. de Kloe, and S. Bartha, "Optimisation of rotating, range-gated fanbeam scatterometer for wind retrieval, Task 3B report: Results and conclusions," Roy. Netherlands Meteorol. Inst., De Bilt, The Netherlands, Tech. Rep. ESA/ESTEC 14383/00/NL/DC, 2003. Accessed: Mar. 23, 2018. [Online]. Available: http:// projects.knmi.nl/scatterometer/rfscat/

[20] A. Stoffelen and M. Portabella, "On Bayesian scatterometer wind inversion," IEEE Trans. Geosci. Remote Sens., vol. 44, no. 6, pp. 1523-1533, Jun. 2006.

[21] T. E. Oliphant and D. G. Long, "Accuracy of scatterometer-derived winds using the Cramér-Rao bound," IEEE Trans. Geosci. Remote Sens., vol. 37, no. 6, pp. 2642-2652, Nov. 1999.

[22] J. Vogelzang, A. Stoffelen, A. Verhoef, J. de Vries, and H. Bonekamp, "Validation of two-dimensional variational ambiguity removal on SeaWinds scatterometer data," J. Atmos. Ocean. Technol., vol. 26, no. 7, pp. 1229-1245, 2009

[23] W. Lin, M. Portabella, A. Stoffelen, J. Vogelzang, and A. Verhoef, "ASCAT wind quality under high subcell wind variability conditions," J. Geophys. Res. Oceans, vol. 120, no. 8, pp. 5804-5819, 2015.

[24] W. Lin and M. Portabella, "Toward an improved wind quality control for RapidScat," IEEE Trans. Geosci. Remote Sens., vol. 55, no. 7, pp. 3922-3930, Jul. 2017.

[25] D. A. Vallado and W. D. McClain, Fundamentals of Astrodynamics and Applications. New York, NY, USA: McGraw-Hill, 1997.

[26] KNMI Scatterometer Team. NSCAT-4 Geophysical Model Function. Accessed: Mar. 22, 2018. [Online]. Available: http://projects.knmi.nl/ scatterometer/nscat_gmf/

[27] M. Portabella and A. Stoffelen, "Scatterometer backscatter uncertainty due to wind variability," IEEE Trans. Geosci. Remote Sens., vol. 44, no. 11 , pp. 3356-3362, Nov. 2006.

[28] J. Vogelzang, A. Stoffelen, A. Verhoef, and J. Figa-Saldaña, "On the quality of high-resolution scatterometer winds," J. Geophys. Res. Oceans, vol. 116, p. C10033, Oct. 2011.

[29] R. D. Lindsley, C. Anderson, J. Figa-Saldaña, and D. G. Long, "A parameterized ASCAT measurement spatial response function," IEEE Trans. Geosci. Remote Sens., vol. 54, no. 8, pp. 4570-4579, Aug. 2016.

[30] M. Portabella and A. Stoffelen, "A probabilistic approach for SeaWinds data assimilation," Quart. J. Roy. Meteorol. Soc., vol. 130, no. 596, pp. 127-152, 2004.

[31] M. H. Freilich and H. Qi, "Scatterometer beam balancing using openocean backscatter measurements," J. Atmos. Ocean. Technol., vol. 16, no. 2, pp. 283-297, 1999.

[32] F. T. Ulaby, R. K. Moore, and A. K. Fung, Microwave Remote Sensing: Radar Remote Sensing and Surface Scattering and Emission Theory, vol. 2. Boston, MA, USA: Addison-Wesley, 1982.

[33] Z. Wang et al., "An SST-dependent Ku-band geophysical model function for RapidScat," J. Geophys. Res. Oceans, vol. 122, no. 4, pp. 3461-3480, 2017.

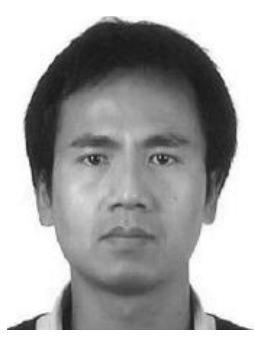

Wenming Lin (M'16-SM'16) was born in Fujian, China, in 1984. He received the B.Sc. degree in engineering from Wuhan University, Wuhan, China, in 2006, and the Ph.D. degree in engineering from the National Space Science Center, Chinese Academy of Sciences, Beijing, China, in 2011.

From 2011 to 2017, he was a Post-Doctoral Researcher at the Institut de Ciències del Mar, Barcelona, Spain, focusing on the advanced oceanographic data processing methods, remote sensing of ocean-surface winds, and data assimilation. $\mathrm{He}$ is currently a Faculty Member with the Nanjing University of Information Science and Technology, Nanjing, China, focusing on ocean remote sensing.

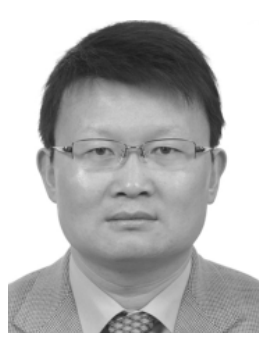

Xiaolong Dong (A'01-SM'01) was born in Shaanxi, China, in 1969. He received the B.E. degree in radio technology and the Ph.D. degree in electromagnetic theory and microwave techniques from Xi'an Jiaotong University, Xi'an, China, in 1991 and 1996, respectively.

From 1996 to 1999, he was a Post-Doctoral Research Fellow and an Associate Professor with the National Space Science Center, Chinese Academy of Sciences, Beijing, China, and promoted as a Full Professor in 1999. From 2001 to 2003, he visited Duke University, Durham, NC, USA. Since 2003, he has been a Hundred Talents Professor of the National Space Science Center with the Chinese Academy of Sciences (CAS), where he is currently the Deputy Director General and the Director with the CAS Key Laboratory of Microwave Remote Sensing. He is the Principal Investigator of projects for the scatterometers of Chinese oceanic observation satellites, including the China-France Oceanic Satellite and the Haiyang-2 satellite prestudy. He has authored more than 80 journal papers and conference presentation. His research interests include theory and techniques of microwave remote sensing and the development of advanced microwave sensors, with emphasis on radar scatterometry for atmospheric, oceanic, and land surface applications.

Dr. Dong has been the Chair of the Microwave Sensors Subgroup of the Working Group on Calibration and Validation of the Committee of Earth Observation Satellites since 2008.

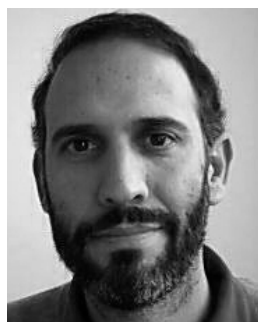

Marcos Portabella was born in Barcelona, Spain, in 1970. He received the B.Sc. degree in physics from the University of Barcelona, Barcelona, in 1994, the M.Sc. degree in remote sensing from the Institute of Space Studies of Catalonia, Catalonia, Spain, in 1995, and the Ph.D. degree in physics from the University of Barcelona in 2002.

$\mathrm{He}$ is currently with the Institut de Ciències del Mar, Barcelona, focusing on satellite remote sensing. In particular, he is involved in scatterometry and L-band radiometry.

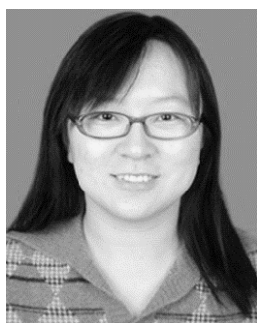

Shuyan Lang was born in Shanxi, China, in 1983. She received the B.E. degree in information engineering from the Beijing Institute of Technology, Beijing, China, in 2004, and the M.S. degree in electromagnetic field and microwave technology from the Chinese Academy of Sciences, Beijing, in 2008.

From 2008 to 2011, she was Research Intern with National Satellite Ocean Application Service, Beijing, and was promoted as an Assistant Research in 2011. Her research interests include the data processing of scatterometer and typhoon monitoring.

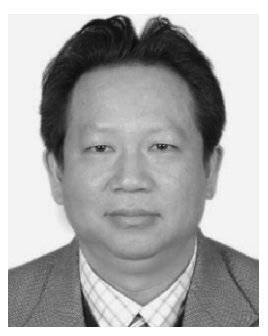

Yijun He (M'03) received the B.S. degree in physics from Hunan Normal University, Changsha, China, in 1985, the M.S. degree in applied physics from Xidian University, Xi'an, China, in 1990, and the $\mathrm{Ph} . \mathrm{D}$. degree in microwave theory and technology from Southeast University, Nanjing, China, in 1993.

$\mathrm{He}$ is currently a Professor of ocean remote sensing and the Dean of the School of Marine Sciences with the Nanjing University of Information Science and Technology, Nanjing, China. He was with the Key Laboratory of Ocean Circulation and Waves, Institute of Oceanology, Chinese Academy of Sciences, Qingdao, China, where he was the Head of the Remote Sensing Group and a Senior Scientist of satellite oceanography from 1996 to 2011. He was a Post-Doctoral Fellow with the Ocean Remote Sensing Institute, Ocean University of China, Qingdao, from 1993 to 1996. He was a Visiting Scientist with the University of Hamburg, Hamburg, Germany, the Bedford Institute of Oceanography, Dartmouth, NS, Canada, and the University of Delaware, Newark, DE, USA. His research interests include ocean waves, sea surface wind speed, and other ocean-surface features related to remote sensing by full polarization synthetic aperture radar and other microwave radar at low incidence angle, air-sea gas exchange using microwave remote sensing and numerical models, sea surface scattering of electromagnetic waves, and mesoscale and submesoscale ocean dynamics. 


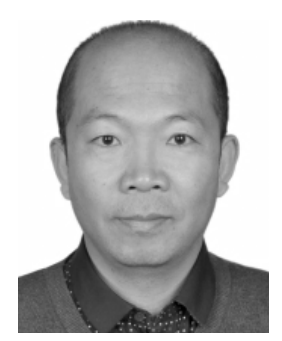

Risheng Yun was born in Inner Mongolia, China, in 1974. He received the B.Sc. degree in computational mathematics from Lanzhou University, Lanzhou, China, in 1995, the M.Sc. degree in engineering from the Northwestern Polytechnical University, Xi' an, China, in 2001, and the Ph.D. degree in engineering from the Institute of Electronics, Chinese Academy of Sciences, Beijing, China, in 2003.

From 2011 to 2012, he was a Visiting Scientist at the Royal Netherlands Meteorological Institute, De Bilt, The Netherlands, focusing on the Numerical Weather Prediction ocean calibration for scatterometers. He is currently a Research Staff with the National Space Science Center, Chinese Academy of Sciences, focusing on scatterometer data processing.

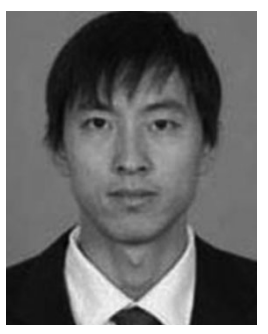

Zhixiong Wang was born in Shaanxi, China, in 1987. He received the master's degree in photogrammetry and remote sensing and the Ph.D. degree in ocean detection technology from the Ocean University of China, Qingdao, China, in 2014 and 2017, respectively.

From 2015 to 2016, he was a joint Ph.D. Student with the Royal Netherlands Meteorological Institute, De Bilt, The Netherlands, with funding from the China Scholarship Council. He is currently a Faculty Member with the Nanjing University of Information Science and Technology, Nanjing, China, focusing on microwave remote sensing and marine physics.

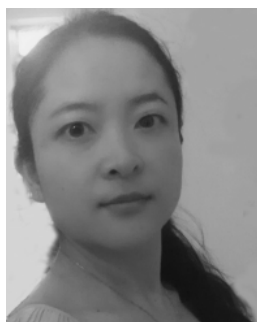

Xingou Xu (M'11) was born in Guizhou, China, in 1984. She received the B.E. degree in surveying engineering from Wuhan University, Wuhan, China, in 2005, the Dual B.A. degree in english from the Huazhong University of Science and Technology, Wuhan, in 2005, and the Ph.D. degree in photogrammetry and remote sensing from Wuhan University in 2011 .

From 2008 to 2009, she was a Visiting Researcher with the University of Edinburg, Edinburg, U.K. From 2011 to 2013, she was a Post-Doctoral Researcher with the National Space Science Center, Chinese Academy of Sciences, Beijing, China, where she is currently an Assistant Professor with the National Space Science Center, focusing on scatterometer data processing and ocean-surface wind field retrieving in different sea status and data simulation.

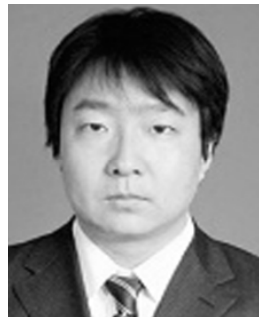

Di Zhu (M'06) was born in Shaanxi, China, in 1978. He received the B.S. degree in communication engineering and the M.S. degree in electromagnetic and microwave engineering from Xi'an Jiaotong University, Xi' an, China, in 2001 and 2004, respectively, and the Ph.D. degree in computer science from the University of Chinese Academy of Sciences, Beijing, China, in 2008.

$\mathrm{He}$ is currently with the National Space Science Center, Chinese Academy of Sciences, Beijing. His research interests include spaceborne and airborne remote sensing payload design and signal processing.

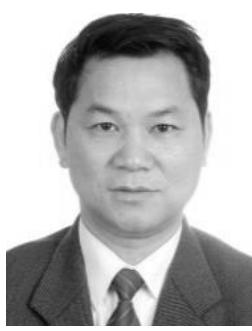

Jianqiang Liu was born in Hunan, China, in 1964. $\mathrm{He}$ received the B.S. degree in atmospheric physics from Peking University, Beijing, China, in 1986, and the M.S. degree in marine meteorological from the National Marine Environmental Forecasting Center (NMEFC), Beijing, in 1989.

From 1989 to 1999, he was with NMEFC, focusing on the ocean remote sensing. He has been a Deputy Director with National Satellite Ocean Application Service, Beijing, since 2010, and he is a Chief Designer of China-France Oceanography Satellite ground segment. His research interests include data preprocessing, satellite application, and sea ice monitoring. 